REVIEW ARTICLE

\author{
R.I. Popa \\ L.A. Gray \\ D.F. Kallmes
}

\section{Urinary Tract Infections in the Potential Vertebroplasty Patient: Incidence, Significance, and Management}

\begin{abstract}
SUMmaRY: Percutaneous vertebroplasty has been deferred due to potential complications from urinary tract infections (UTIs). Our aim was to help sort out the types of treatment needed for percutaneous vertebroplasty patients and the length delay for performing the procedure. We searched for information regarding the ramifications of infection from the literature and devised a plan of treatment for the various infectious problems that may occur in patients undergoing percutaneous vertebroplasty. We devised a plan of action for the screening, testing, diagnosis and treatment of patients with a potential UTI who are presenting for a percutaneous vertebroplasty
\end{abstract}

0 ne of the most feared potential complications from vertebroplasty is that of infection of the cement implant. Fortunately, the reported rate of localized infection of the implant is rare, with fewer than 10 reported cases. ${ }^{1-5}$ The ramifications of such infection are severe because clearance of the infection without vertebrectomy would be unlikely. Efforts to avoid cement infection have been previously described in the vertebroplasty literature, including routine use of either systemic or local infusion of prophylactic antibiotics as well as exclusion of pre-existing infections either in the target vertebral body or elsewhere. ${ }^{6}$

In the context of dire consequences from untoward infection of the cement implant, to our knowledge, the issues surrounding the management of urinary tract infection (UTI) have received scant prior attention in regard to planned vertebroplasty procedures. The population typical of consideration for vertebroplasty, female septa- or octogenarians, also has high rates of urinary tract abnormalities. These abnormalities range from asymptomatic pyuria or bacteriuria to overt infection. The significance of and management decisions for these signs and symptoms, especially in the setting of vertebroplasty, very likely are not well understood by most practicing radiologists.

The purpose of the current article was to review our experience regarding the spectrum of UTI in patients presenting for potential vertebroplasty. We also briefly review the literature regarding infectious complications in vertebroplasty, to uncover whether such reported complications might advise management decisions. Further, we offer a brief review of UTI in the elderly, including symptoms, diagnostic testing, and therapy. Finally, we offer practical recommendations for vertebroplasty practitioners.

\section{Infectious Complications Reported in Vertebroplasty}

We reviewed the literature from April 2004 to May 2008 and identified fewer than 10 cases of infectious complications from vertebroplasty. ${ }^{1-5}$ In most of these reported cases, a specific infectious agent was not identified. The bacteria isolated in

From the Departments of Hospital Internal Medicine (R.I.P.) and Radiology (L.A.G., D.F.K.), Mayo Clinic, Rochester, Minn.

Please address correspondence to Leigh A. Gray, MD, Department of Radiology, Mayo Clinic, 200 First St SW, Rochester, MN 55905; e-mail: gray.leigh@mayo.edu

Indicates open access to non-subscribers at www.ajnr.org

DOI 10.3174/ajnr.A1385 other reports were Serratia marcescens, Stenothrophomonas maltophilia, Burkholderia cepacia, and Streptococcus agalactiae. ${ }^{3}$ However, even in these latter cases in which specific bacteria were identified, the sources remained unknown. In at least 1 of these articles, the patient had a UTI diagnosed and treated before the procedure. ${ }^{5}$ It is not clear if there was any association between the UTI and the reported infection in that case report.

Intuitively, 2 potential sources of infectious agents should be considered, including the skin that is entered during the procedure as well as active bacteremia during or after cement infusion. With the current practice of skin preparation before procedures and more recent widespread use of chlorhexidine, skin infections after invasive procedures have decreased significantly. To mitigate the probability of blood-borne agents infecting the cement implant, one may need to address systemic infections with a risk for bacteremia that can increase the risk for infectious complications after vertebroplasty. UTI represents 1 of these infections with a risk of bacteremia.

\section{Bacteremia with UTI}

At least intuitively, it would be unwise from the perspective of infectious complications postoperatively to perform an invasive procedure on a patient with bacteremia, especially a procedure that involves a permanent implant. In a study that analyzed community-acquired bacteremias, ${ }^{7}$ UTIs were the most commonly identified source of bacteremia, responsible for $29 \%$ of the community-acquired bacteremias with a single identifiable source. Escherichia coli were identified in 15\% of cases of bacteremia. Bacteria commonly causing UTIs were the agent for at least $30 \%$ of the cases of community-acquired bacteremia. Patients older than 65 years of age were significantly more likely to have a urinary tract source, and $44 \%$ of these elderly patients had indwelling urinary catheters. In another retrospective study that included 350 patients with symptomatic UTI, 53 (15\%) were bacteremic. ${ }^{8}$ The most common pathogens isolated from this latter series of patients with bacteremia and UTIs were E coli (40\% of episodes), Klebsiella species (17\%), Proteus mirabilis (17\%), and enterococci (14\%).

Five variables were independently associated with bacteremia in the setting of UTI: residence at home rather than in an institution (odds ratio [OR] 4; 95\% confidence interval [CI], 1.5-10.7); presence of an indwelling urinary catheter (OR 3.3; 95\% CI, 1.3-8.8); presence of band forms in the blood count 
Table 1: Spectrum of abnormal urinalysis findings in patients presenting for vertebroplasty at our institution

\begin{tabular}{ll}
\hline & No. $(\%)$ \\
\hline Total No. of patients & 30 \\
Women & $25(83 \%)$ \\
Average age (yr) & 65.6 \\
Positive Gram stains & $17(56.6 \%)$ \\
Positive urine cultures & $6(20 \%)$ \\
Positive bacteria on U/A & $18(60 \%)$ \\
Patients treated with antibiotics & $23(76.6 \%)$ \\
Follow-up urine after antibiotics & $1(3.3 \%)$ \\
PV postponed due to UTI & $10(33.3 \%)$ \\
\hline
\end{tabular}

Note:-U/A indicates urine analysis; PV, percutaneous vertebroplasty; UTI, urinary tract infection.

(OR 3.3; 95\% CI, 1.5-7.2); shaking chills (OR 2.3; 95\% CI, 1.1-4.8); neutrophilia (OR 1.1; 95\% CI, 1.04-1.15).

The above data ${ }^{8}$ regarding bacteremia are from studies that included patients ill enough to present to the emergency department and to warrant evaluations that included blood cultures. Common practice is to perform blood cultures for more severe cases of UTI. Thus, the incidence of bacteremia in patients presenting for vertebroplasty is most likely substantially lower than that in the above-mentioned series. ${ }^{7}$

\section{Incidence of UTI in the Vertebroplasty Population}

After approval by our local institutional review board, we reviewed the medical records of 735 consecutive patients presenting for vertebroplasty evaluation between April 2004 and January 2008. Some of these patients were included in prior publications from our institution, but we have never previously evaluated the incidence of UTIs in this population. ${ }^{9,10}$ We identified $30(4.1 \%)$ patients who had either symptoms referable to the urinary tract, including dysuria, or a recent abnormal urinalysis result before planned vertebroplasty. Because this was a retrospective analysis and no standardized screening for UTI was performed, there may have been more patients with the same characteristics who were not identified before vertebroplasty. Summary data are shown in Table 1. These results suggest that the diagnostic tests and criteria for UTI were heterogeneous. Some patients underwent their vertebroplasty before completion of treatment and not all patients were treated for UTI.

\section{Periprocedural Management of UTIs in Other Surgical Specialties}

Urinary tract and other types of infections are a concern before any invasive procedure. The only surgical subspecialty with a standardized periprocedural management approach in this matter is urology. ${ }^{11,12}$ Patients scheduled for a procedure involving the urinary tract, such as transurethral resection of the prostate or other procedures with potential compromise of the urinary tract mucosa, are tested for bacteriuria, regardless of symptoms. The presence of bacteriuria mandates antibiotic therapy before the urologic procedure. If the procedure is not urgent, antibiotic treatment is completed and a follow-up urine study, either a Gram stain or culture, is performed. If the procedure cannot be postponed to complete the treatment of a UTI, antibiotics are started before the intervention and continued to complete the course postoperatively.

An increasing number of orthopedic groups are actively screening for UTI, and some groups recommend full antibiotic treatment for patients diagnosed with UTI before arthroplasties. This approach was dictated by increasing evidence that UTIs and other infections can cause infectious complications after joint replacement.

\section{Diagnosis and Management of UTIs in the Elderly}

UTI is the most common community-acquired bacterial infection, accounting for 7 million office visits, 1 million emergency department visits, and approximately 100,000 hospitalizations each year in the United States. ${ }^{5}$ UTIs account for $25 \%$ of community-acquired bacterial infections and $30 \%$ of bacterial infections in the elderly in nursing homes. ${ }^{13,14}$

\section{Clinical Presentation}

Three clinical entities will be the focus of this review: UTI, pyelonephritis, and asymptomatic bacteriuria. Classic symptoms of UTI are dysuria, urinary frequency, hematuria, urgency, and suprapubic discomfort. The probability of a UTI in a patient presenting with 1 or more symptoms of UTI is approximately $50 \%$. The probability of UTI increases to $96 \%$ if the patient presented with the triad of dysuria, frequency, and no vaginal symptoms. Vaginal infections can mimic some of the UTI symptoms and can influence urine testing, especially clean-catch samples.

Geriatric patients represent a large subgroup of high-risk patients for UTI. The elderly have an altered immune system and may fail to demonstrate traditional clinical indicators of infection. In the geriatric population, symptoms like nausea, vomiting, decreased urinary output, and change in mental status may represent manifestations of a UTI. This elderly cohort of patients has fewer systemic symptoms than younger patients, and some of the local symptoms may be obscured by pre-existing chronic genitourinary symptoms, including urinary incontinence, neurogenic bladder, and benign prostatic hypertrophy. For these reasons, the diagnosis of UTI, and especially bacteremic UTI, is often delayed in the elderly patient.

Acute pyelonephritis represents an infection of the upper urinary tract. Acute pyelonephritis is a clinical syndrome characterized by fever, chills, and flank pain or tenderness, usually accompanied by symptoms of a lower UTI, including urinary frequency, urgency, and dysuria. These patients are subsequently found to have bacteriuria. The diagnostic approach may include additional imaging of the urinary tract, including CT or sonography, especially if patients do not respond to treatment after 3 days. ${ }^{15,16}$

\section{Laboratory Evaluation}

The laboratory diagnosis of UTI includes urinalysis by dipstick, urine microscopy, and the urine Gram stain and culture; and these are detailed below:

Urinalysis by Dipstick. For patients who manifest at least 1 symptom, a positive leukocyte esterase has a sensitivity of $75 \%-96 \%$ and a specificity of $94 \%-98 \%$ for detection of UTI. Nitrite is less useful because it is positive only for bacteria that produce nitrate reductase and can be falsely positive with consumption of ascorbic acid.

Urine Microscopy. When a cutoff of more than 6 white blood cells per high-power field is used, the sensitivity and specificity of urine microscopy is $82 \%-97 \%$ and $84 \%-95 \%$, 


\begin{tabular}{l}
\hline Table 2: Complicating factors of UTIs \\
\hline Complicating Factors \\
\hline History \\
Diabetes \\
Pregnancy \\
Immunosuppressed (eg, steroids, chemotherapy) \\
Renal calculi or renal insufficiency \\
Known functional or structural urologic abnormalities \\
Urinary tract catheterization (or other urologic procedure or \\
instrumentation) within last 2 weeks \\
Discharge from hospital or nursing home within last 2 weeks \\
$\geq 4$ UTIs within last 12 months \\
Failure of TMP/SMX treatment for UTI within last 4 weeks \\
Resident of extended-care facility \\
Symptoms \\
$>7$ Days duration \\
Rigors (shaking chills) \\
Flank pain: midback, severe, new, occurring with onset of these symptoms \\
Temperature $>101^{\circ} \mathrm{F}$ \\
\hline
\end{tabular}

Note:-TMP indicates trimethoprim; SMX, sulfamethoxazole.

respectively, for diagnosis of UTI, when symptoms are present.

Urine Culture. The sensitivity of urine culture depends on the threshold used for defining a positive test. When using a threshold of $>100$ colony-forming units (CFUs)/mL, urine culture has a sensitivity of approximately $90 \%$; sensitivity decreases to $50 \%$ for a threshold of $>100,000$ colonies $/ \mathrm{mL}$. The specificity of the test ranges from $85 \%$ to $99 \%$. Urine culture results are usually available in $24-48$ hours. Microbial etiology is dominated by E Coli (80\%), followed by Staphylococcus saprophyticus (15\%) and other Enterobacteriaceae. These percentages may change in complicated UTIs. Polymicrobial growth suggests inappropriate collection.

The method of collection for the urine sample can influence the results. Apparent pyuria and bacteriuria in a cleancatch sample may represent vaginal contamination; therefore, ruling out vaginal infections is important before interpreting urine test results. Some authorities suggest a threshold of $100,000 \mathrm{CFU} / \mathrm{mL}$ for clean-catch samples versus $100 \mathrm{CFU} / \mathrm{mL}$ for catheterized samples.

\section{Complicating Factors}

A complete diagnosis of UTI should include an evaluation for complicating factors (Table 2). The presence of complicating factors requires a different diagnostic and therapeutic approach. ${ }^{8,13}$ Urine culture and sensitivity should be obtained before initiation of treatment, and a urine culture should be obtained at the completion of an antibiotic course.

\section{Asymptomatic Bacteriuria}

The diagnosis of asymptomatic bacteriuria should be based on results of the culture of a urine specimen collected in a manner that minimizes contamination. For asymptomatic women, bacteriuria is defined as 2 consecutive voided urine specimens with isolation of the same bacterial strain in quantitative counts, $>100,000 \mathrm{CFU} / \mathrm{mL}$. For asymptomatic men, bacteriuria is defined as a single clean-catch voided urine specimen with 1 bacterial species isolated in a quantitative count, $>100,000 \mathrm{CFU} / \mathrm{mL}$. A single catheterized urine specimen with 1 bacterial species isolated in a quantitative count of $>100$ $\mathrm{CFU} / \mathrm{mL}$ identifies bacteriuria in both women and men. ${ }^{9}$

\begin{tabular}{lc}
\hline $\begin{array}{l}\text { Table 3: Prevalence of asymptomatic bacteriuria in selected } \\
\text { populations }\end{array}$ \\
\hline Patient population & Prevalence (\%) \\
\hline Healthy premenopausal women & $1.0-5.0$ \\
Pregnant women & $1.9-9.5$ \\
Postmenopausal women 50-70 years of age & $2.8-8.6$ \\
Patients with diabetes & \\
$\quad$ Women & $9.0-27$ \\
$\quad$ Men & $0.7-11$ \\
Elderly persons in the community & $10.8-16$ \\
$\quad$ Women & $3.6-19$ \\
$\quad$ Men & \\
Elderly persons in a long-term-care facility & $25-50$ \\
$\quad$ Women & $15-40$ \\
$\quad$ Men & \\
Patients with spinal cord injuries & $23-89$ \\
$\quad$ Intermittent catheter use & 57 \\
$\quad$ Sphincterotomy and condom catheter in place & 28 \\
Patients undergoing hemodialysis & \\
Patients with indwelling catheter use & $9-23$ \\
$\quad$ Short-term & 100 \\
\hline
\end{tabular}

Asymptomatic bacteriuria is a common finding, and its prevalence depends on the subgroup of the population studied (Table 3). ${ }^{11,15}$ The microbiology of asymptomatic bacteriuria is not significantly different from the microbiology of symptomatic UTI, with $E$ Coli accounting for $75 \%-80 \%$ of cases, followed by $S$ saprophyticus and other Enterobacteriaceae such as Proteus and Pseudomonas species.

There is enough evidence to make recommendations regarding asymptomatic bacteriuria for several subgroups of patients, including pregnant women and patients scheduled for urologic procedures. ${ }^{11}$ As noted previously, screening for and treatment of asymptomatic bacteriuria is recommended before transurethral resection of the prostate and other urologic procedures for which mucosal bleeding is anticipated. Conversely, screening for or treatment of asymptomatic bacteriuria is not recommended for the following categories of asymptomatic patients ${ }^{17}$ : premenopausal nonpregnant women; women with diabetes; older persons living in the community; elderly institutionalized subjects; persons with spinal cord injury; catheterized patients while the catheter remains in situ.

Pyuria accompanying asymptomatic bacteriuria is not an indication for antimicrobial treatment. Antimicrobial treatment of asymptomatic women with catheter-acquired bacteriuria that persists 48 hours after indwelling catheter removal may be considered.

\section{Treatment of UTIs}

The treatment for uncomplicated UTIs includes trimethoprim/sulfamethoxazole for 3 days as a first-line therapy. ${ }^{18,19}$ Other options are trimethoprim alone for 3 days or fluoroquinolone (ofloxacin, norfloxacin, ciprofloxacin) for 3 days. Nitrofurantoin for 5-7 days or cefpodoxime for 3 days may also be used. The duration of treatment for acute pyelonephritis is 7-14 days, and a higher percentage of patients will require hospitalization than for the lower UTI group. For complicated UTIs, the treatment is usually started with trimethoprim/sulfamethoxazole or fluoro- 


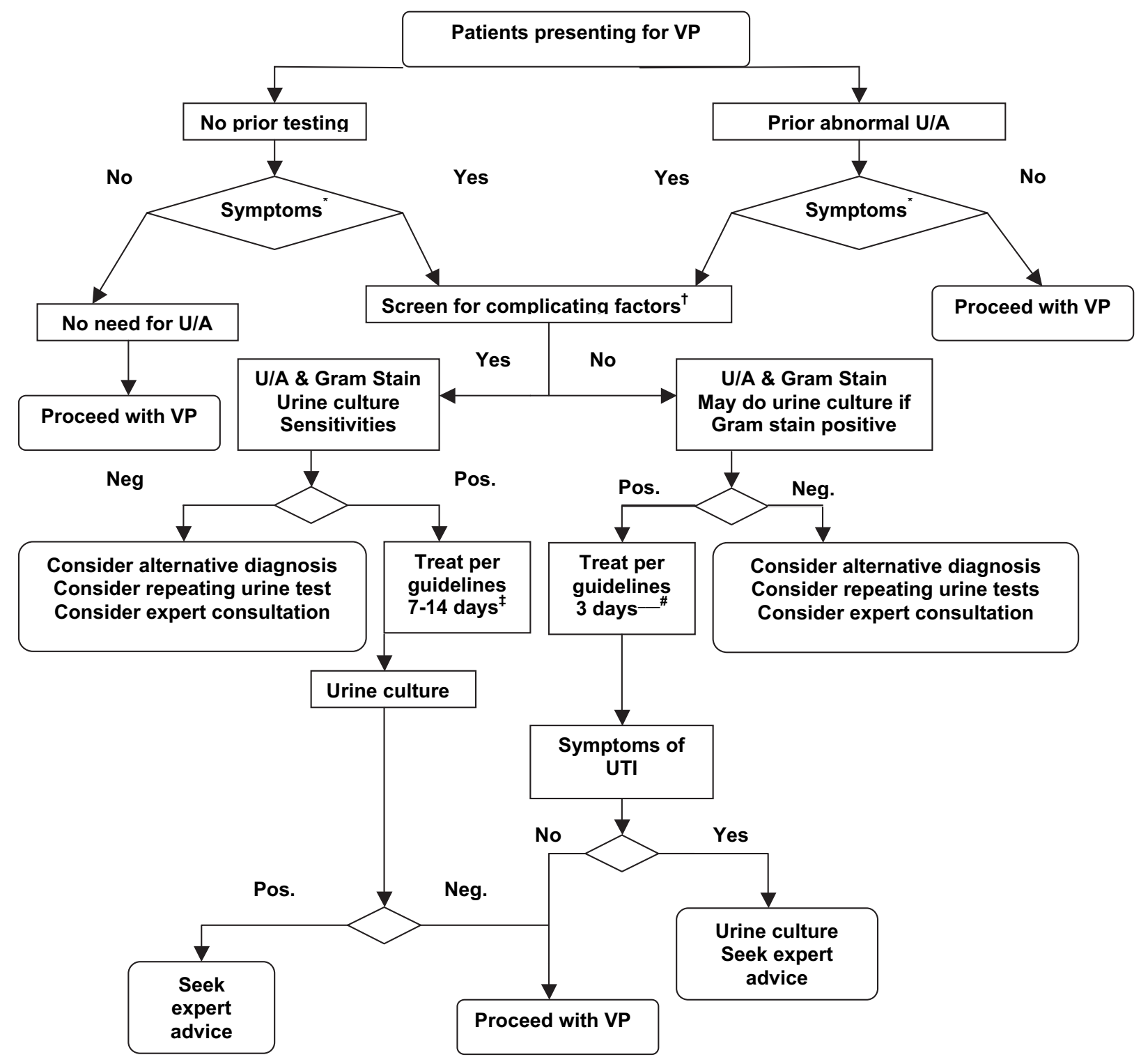

Fig 1. Algorithm for evaluation of patients considered for vertebroplasty (VP). *Dysuria, urine frequency, hematuria, urgency, and suprapubic discomfort. See text for UTI symptoms in the geriatric population. †See Table 2. ¥Start treatment with trimethoprim/sulfamethoxazole 160/800 twice a day (BID) or fluoroquinolone and be guided by sensitivities obtained from urine culture. The duration of treatment is 7-14 days. §Trimethoprim/sulfamethoxazole 160/800 BID is the first-line treatment. Trimethoprim alone, fluoroquinolones for 3 days, or nitrofurantoin for $5-7$ days is an alternative option. U/A indicates urine analysis; Pos., positive; Neg, negative.

quinolone and is guided by sensitivities obtained from the urine culture. The duration of treatment is $7-14$ days. Follow-up urine testing is not recommended for patients treated for uncomplicated UTIs that are asymptomatic after treatment. Obtaining follow-up to document clearing of the urine for patients with complicated UTI is recommended. The local patterns of resistance to antibiotics may play a role in selecting the choice for the empiric therapy. For example, if $>20 \%$ of the isolates at your local laboratory are resistant to trimethoprim/sulfamethoxazole, another antibiotic like fluoroquinolone should be the first choice for treatment of an UTI.

\section{Recommendations for Patients Being Considered for Vertebroplasty}

The diagnosis and management of patients presenting for vertebroplasty with suggested UTI is outlined in Fig 1.
Screening for Symptoms. All patients referred for vertebroplasty should be screened for symptoms of UTI, including dysuria, urinary frequency, hematuria, urgency, and suprapubic discomfort. In the geriatric population, symptoms like nausea, vomiting, decreased urinary output, or change in mental status may represent manifestations of a UTI. Patients without signs or symptoms to suggest UTI do not need to undergo preprocedural urinalysis.

The presence of these symptoms should prompt testing, as described in the next section. Special attention should be given to the duration of symptoms, presence of fever, chills, laboratory abnormalities such as neutrophilia and altered renal function, as well as other complicating factors (Table 2).

Testing for UTI. Patients presenting for consideration of vertebroplasty who have symptoms referable to the urinary tract, as outlined in the preceding paragraph, should undergo 
a urinalysis with a Gram stain. If there is no suggestion of vaginitis, a clean-catch specimen is acceptable. If there are suggestions of vaginal infection, not only should this be evaluated by a gynecologist but also a catheterized urine sample is advised. For patients who may have difficulty in cooperating with proper specimen collection, such as patients with neurologic conditions, immobilized patients, or patients of advanced age, we recommend obtaining a catheterized sample. Urine culture may be obtained if results of the Gram stain are abnormal, and we recommend asking the laboratory to hold the sample for culture. If complicating factors listed in Table 2 are present, then urine culture is mandatory.

A limited number of patients may present for vertebroplasty with abnormal urine tests that were obtained by other providers. The same algorithm as shown in Fig 1 applies to these patients. Screening for symptoms of UTI is the first step in that decision tree. Asymptomatic bacteriuria and asymptomatic pyuria in the vertebroplasty patient are not indications for additional testing or treatment. If symptoms are present, treatment can be started after screening for complicating factors, without additional testing, following the same guidelines. A urine culture should be obtained if complicating factors are present.

Another special subgroup is patients who were already treated for UTI before presenting for vertebroplasty. The algorithm presented in Fig 1 applies to them as well. Follow-up testing will be decided on the basis of the presence of symptoms after treatment and the presence of complicating factors.

Diagnosis of UTI. Symptomatic patients with positive leukocyte esterase tests, $>6$ white blood cells per high-power field or abnormal results of Gram stains, very likely have a UTI and require treatment. Additional testing may be needed for a UTI with complicating factors, and the work-up should be decided in conjunction with a general practitioner and/or urologist. Sensitivities and specificities of various tests are laboratory-specific; therefore, clinicians should know their laboratory-specific likelihood ratios.

Treatment. Three days of treatment with trimethoprim/ sulfamethoxazole or a quinolone for patients with uncomplicated UTIs are recommended. Seven to 14 days' duration of therapy is indicated for complicated UTIs. The choice of antibiotic should be revisited after culture and sensitivities become available.

Follow-Up Testing. No follow-up urine testing is needed for patients who are asymptomatic after completion of treat- ment. Urine culture, or at least a Gram stain, is recommended for complicated UTIs following treatment or if the patient continues to be symptomatic at completion of treatment.

\section{Acknowledgment}

We thank Arash Ehteshami Rad, MD, for all the help in the publication of this article.

\section{References}

1. Alfonso Olmos M, Silva Gonzalez A, Duart Clemente J, et al. Infected vertebroplasty due to uncommon bacteria solved surgically: a rare and threatening life complication of a common procedure-report of a case and a review of the literature. Spine 2006;31:E770-73

2. Schmid KE, Boszczyk BM, Bierschneider M, et al. Spondylitis following vertebroplasty: a case report. Eur Spine J 2005;14:895-99

3. Vats HS, McKiernan FE. Infected vertebroplasty: case report and review of literature. Spine 2006;31:E859-62

4. Walker DH, Mummaneni P, Rodts GE Jr. Infected vertebroplasty: report of two cases and review of the literature. Neurosurg Focus 2004;17:E6

5. Yu SW, Chen WJ, Lin WC, et al. Serious pyogenic spondylitis following vertebroplasty: a case report. Spine 2004;29:E209-11

6. Hernandez L, Munoz ME, Goni I, et al. New injectable and radiopaque antibiotic loaded acrylic bone cements. J Biomed Mater Res B Appl Biomater 2008; 87B:312-20. [Epub ahead of print]

7. Lark RL, Saint S, Chenoweth C, et al. Four-year prospective evaluation of community-acquired bacteremia: epidemiology, microbiology, and patient outcome. Diagn Microbiol Infect Dis 2001;41:15-22

8. Bahagon Y, Raveh D, Schlesinger Y, et al. Prevalence and predictive features of bacteremic urinary tract infection in emergency department patients. Eur J Clin Microbiol Infect Dis 2007;26:349-52

9. Gray LA, Jarvik JG, Heagerty PJ, et al. Investigational vertebroplasty efficacy and safety trial (INVEST): a randomized controlled trial of percutaneous vertebroplasty. BMC Musculoskelet Disord 2007;8:126

10. Rad AE, Kallmes DF. Pain relief following vertebroplasty in patients with and without localizing tenderness on palpation. AJNR Am J Neuroradiol. 2008;29: 1622-26. Epub 2008 Jun 26

11. Wolf JS Jr, Bennett CJ, Dmochowski RR, et al. Best practice policy statement on urologic surgery antimicrobial prophylaxis. J Urol 2008;179:1379-90

12. Terris MK. Recommendations for prophylactic antibiotic use in genitourinary surgery. Contemporary Urology 2001:12-27

13. Juthani-Mehta M. Asymptomatic bacteriuria and urinary tract infection in older adults. Clin Geriatr Med 2007;23:585-94

14. Mouton CP, Bazaldua OV, Pierce B, et al. Common infections in older adults. Health Care Food Nutr Focus 2001;18:1, 3-7

15. Ramakrishnan K, Scheid DC. Diagnosis and management of acute pyelonephritis in adults. Am Fam Physician 2005;71:933-42

16. Sandler CM, Choyke PL, Bluth E, et al. Acute pyelonephritis. Available at: $\mathrm{http} / /$ www.guideline.gov/summary/summary.aspx?ss $=15 \&$ doc_id $=8277 \&$ $\mathrm{nbr}=4609$. Accessed April 25, 2008

17. U.S. Preventive Services Task Force. Screening for asymptomatic bacteriuria in adults: U.S. Preventive Services Task Force reaffirmation recommendation statement. Ann Intern Med 2008;149:43-47

18. Nicolle LE. Uncomplicated urinary tract infection in adults including uncomplicated pyelonephritis. Urol Clin North Am 2008;35:1-12

19. Warren JW, Abrutyn E, Hebel JR, et al. Guidelines for antimicrobial treatment of uncomplicated acute bacterial cystitis and acute pyelonephritis in women: Infectious Diseases Society of America (IDSA). Clin Infect Dis 1999;29:745-58 